\title{
Anal Intercourse and HIV Risk Among Low-Income Heterosexual Women: Findings from Chicago HIV Behavioral Surveillance
}

\author{
Britt S. Livak* , Nikhil G. Prachand and Nanette Benbow \\ Chicago Department of Public Health, STI/HIV Division, 333 South State Street, Suite200, Chicago, IL60604, USA
}

\begin{abstract}
Background: Anal intercourse (AI) is a highly efficient route for HIV transmission and has not been well elucidated among heterosexual (HET) women. Heterosexual women living in impoverished urban areas in the US are at increased risk for HIV acquisition. We aim to describe rates of AI and characteristics associated with AI among heterosexual women at increased risk for HIV acquisition living in Chicago.

Methods: The Chicago Department of Public Health conducted a survey of HET during 2007 as part of the National HIV Behavioral Surveillance System. Venue-based, time-location sampling was used to select participants from venues in high-risk areas (census tracts with concurrently high rates of heterosexual AIDS and household poverty). Eligible participants were interviewed anonymously and offered a HIV test.

Results: In total, 407 heterosexual women were interviewed. Seventy-one (17\%) women reported having AI in the past 12 months, with 61 of the $71(86 \%)$ reporting unprotected AI. In multivariate analysis, women who engaged in AI were more than three times as likely to have three or more sex partners in the past 12 months (OR=3.27, 95\% CI 1.53-6.99). AI was also independently associated with STI diagnosis in the past 12 months (2.13, 95\% CI 1.06-4.26), and having sexual intercourse for the first time before the age of 15 years $(2.23,95 \%$ CI 1.28-3.89).

Conclusion: AI was associated with multiple high risk behaviors including a greater number of sexual partners, STI diagnosis, and earlier age at first sex. The combination of risk factors found to be associated with AI call for new HIV prevention services tailored to the needs of women and young girls living in poverty.
\end{abstract}

Keywords: HIV/AIDS, heterosexual, anal intercourse, women, behavioral surveillance.

\section{INTRODUCTION}

Human Immunodeficiency Virus (HIV) prevalence among heterosexuals living in high-poverty urban areas in the United States (U.S.) has recently been described by the Centers for Disease Control and Prevention (CDC) as a generalized epidemic [1]. Heterosexual women are more heavily impacted by HIV infection than heterosexual men. In 2008 , women accounted for $66 \%$ of heterosexual HIV diagnoses in the U.S., and $76 \%$ of heterosexual HIV diagnoses in Chicago [2,3].

Anal intercourse (AI) is often overlooked as a risk factor for HIV acquisition among heterosexuals, yet a substantial proportion of heterosexual women report participating in AI in their lifetime. Approximately $34 \%$ of women from a national probability sample aged 20-44 reported ever having AI [4], and it is estimated that approximately $9 \%$ of women in the general population have practiced AI in the past 12 months [5].

AI is a highly efficient route for HIV transmission. The estimated risk per act for acquiring HIV is 5 times greater for participation in receptive AI compared with receptive vaginal intercourse [6]. Additionally, condom use is reported

*Address correspondence to this author at the Chicago Department of Public Health, STI/HIV Division, 333 South State Street, Suite200, Chicago, IL60604, USA; Tel: 312-747-9546; Fax: 312-745-3923;

E-mail: britt@informationstationllc.com less frequently with anal intercourse than it is with vaginal intercourse [7].

Several studies have examined factors associated with heterosexual AI. However, much of the focus has been on women recruited from injection drug using (IDU), or sexually transmitted infection (STI) clinic populations. Among women attending public STI clinics, AI has been associated with exchanging sex for money or drugs [8,9], substance use [9], sex on the same day as meeting a partner [9], having more than three sex partners in one's lifetime [9], and non AfricanAmerican race/ethnicity [9]. Among female IDUs, AI has been associated with exchange sex [10, 11], bisexuality [10], amphetamine use [11], and sex with partners who were nonheterosexual or injection drug users [10].

The proportion of people living with AIDS in the U.S. is increasing among women and among the poor [12]. A study by Peterman et al. found that the counties in the U.S. with the largest increases in incident AIDS cases had higher proportions of low-income households and substandard social conditions than the counties in the U.S. with the smallest increases in incident AIDS cases [13].

The noted increase in AIDS cases among women living in poverty underscores the need for an understanding of the HIV risk factor dynamics in this group. Previous research has only assessed factors associated with AI among specific subgroups of heterosexual women. Little is known about factors related to AI among this particularly vulnerable group in Chicago. The aim of this paper, therefore, is to 
describe rates of $\mathrm{AI}$ and characteristics of heterosexual women who engage in AI, among a representative sample of women living in low-income areas of Chicago.

\section{MATERIALS AND METHODS}

\section{Study Design}

The Chicago Department of Public Health conducted a survey of heterosexual males and females between March and October of 2007 as part of the National HIV Behavioral Surveillance system (NHBS). NHBS is an ongoing CDC funded project that collects cross-sectional data on HIV/STI risk behaviors, HIV testing behaviors, and exposure to HIV prevention in populations that are at-risk for HIV infection. NHBS is conducted in 21 cities in three populations: Men Who Have Sex With Men (MSM), Injection Drug Users (IDU), and Heterosexuals living in High Risk Areas (HET). Surveys are conducted annually, and repeated in three year cycles. Participants were eligible if they were between 18 and 50 years of age, residents of Chicago's metropolitan statistical area, and if they reported vaginal or anal intercourse with a member of the opposite sex in the past 12 months.

The National Center for HIV, STD, and TB Prevention (NCHSTP), CDC, has determined that NHBS is a public health surveillance activity, used for disease control program or policy purposes and is not considered to be research. Therefore, the CDC Human Subjects Protection Office made a determination that the CDC was "not engaged" in the research and thus NHBS protocol did not require CDC IRB review. All local human subject research activities were approved by the Chicago Department of Public Health Institutional Review Board. Verbal consent was secured and recorded from each research participant for the survey component. Standard written consent was used for the HIV testing component.

\section{Sampling Methods}

Venue-based, time-location sampling (VBS) was used to select participants from venues in high-risk areas (HRAs) of Chicago. VBS is a three stage process. First, formative research is conducted to identify potential venues for sampling and learn about the sample population. The second stage involves creating a sampling frame which includes venues and the venue day-time periods (VDT) when it is possible to conduct sampling. Venues and VDTs are randomly selected in two stages to create monthly sampling calendars. The third stage of VBS entails randomly selecting and interviewing participants within the venues [14].

Formative research was conducted between February 2006 and February 2007. During formative research, HRAs were identified using CDC-NHBS protocol. HRAs are census tracts with high rates of AIDS cases attributed to heterosexual contact, and census tracts with high rates of poverty. Chicago 2004 AIDS Case surveillance data and 2000 U.S. census data were used to define these areas. Census tracts were ranked according to the areas with the highest rates of both AIDS and poverty. The top 15\% (123) of ranked census tracts were chosen for investigation.

The 123 census tracts were observed noting population density, abundance of potential venues for recruitment, pedestrian activity, sampling feasibility, and signs of gentrification. Gentrified census tracts were excluded from sampling.

A total of 15 geographically diverse census tracts were determined to be HRAs in Chicago. Respondents were sampled from 74 venues within these census tracts. The venues included convenience stores, laundromats, street corridors, beauty salons, churches, and other locations.

Heterosexuals attending venues were randomly approached and were asked if they were interested in participating and undergoing eligibility screening. Participants were administered an anonymous, face-to-face, standardized questionnaire using a hand-held computer. Eligible participants were also offered HIV testing using Orasure $^{\circledR}$ testing technology (Orasure Technologies Inc, Bethlehem, PA, USA).

The sample size goal designated by the CDC was 750 participants. NHBS-Chicago recruited 851 eligible participants; 412 of which were women. Participants were compensated up to $\$ 50$ for the time and effort required for the questionnaire and HIV test.

\section{Measures}

The standardized questionnaire gathered data about respondents' sexual and drug-use behaviors, HIV and STI testing behaviors, and exposure to and utilization of HIV prevention services. All of the information obtained in the survey was self-reported.

\section{Sexual Risk Behaviors}

Participants were asked about their sexual behavior within the 12 months prior to the survey. They were asked about the types of partners they had, the types of sex they had with each partner type, whether or not condoms were used during sex, the number of sexual partners they had in the past 12 months, and the age at which they first initiated vaginal or anal intercourse. Three partner types were assessed in the survey: main, casual, and exchange. These partner types were defined as follows: "Main partners are partners you have sex with who you feel committed to above anyone else (includes girlfriend, wife, significant other or life partner); casual partners are partners you have sex with but do not feel committed to or don't know very well; and exchange partners are partners you have sex with in exchange for things like money or drugs".

Likewise, three types of sexual intercourse were assessed in the survey: oral, vaginal, and anal. The three types were defined as follows: "Oral sex means mouth on the vagina or penis; vaginal sex means penis in the vagina; and anal sex means penis in the anus or butt". Concurrency was measured by asking participants whether or not they had sex with "other people" during the time of their most recent sexual relationship.

\section{Drug-Use Behaviors}

Participants were asked whether or not they used any "non-injection" drugs that were not prescribed to them in the past 12 months. They were also asked about their participation in "binge drinking" in the past 12 months. "Binge drinking" for a female is defined as having 4 or more drinks in one sitting [15]. The frequency of binge drinking 
was assessed by asking the female respondent, "In the past 12 months, how often did you have 4 or more alcoholic drinks in one sitting?"

\section{HIV/STI Testing Behaviors and Exposure to HIV Prevention}

To assess whether or not participants tested for HIV on a regular basis, the survey asked how many times participants had been tested for HIV in the previous two years. The survey also asked whether or not participants were exposed to and utilized HIV prevention services by asking if any of the following had occurred in the previous 12 months: they received any free condoms from someone other than a friend, relative, or sex partner, or they participated in any individual-level or group-level HIV interventions. Participants also reported whether they had been diagnosed with any STIs in the past 12 months.

\section{Other Variables}

Additional variables included in the analysis were: homelessness in the past 12 months, defined as: "living on the street, in a shelter, a Single Room Occupancy hotel (SRO), temporarily staying with friends or relatives, or living in a car," current employment status, estimated annual income (annual household income divided by the number of people dependent on the income), sexual identity (heterosexual, bisexual, or homosexual), and being arrested and detained for over 24 hours in the past 12 months.

\section{Statistical Analysis}

The outcome for analysis was receptive anal intercourse, defined as participation in AI with a partner of any type in the past 12 months. Data were limited to female participants who had no history of injection drug use in the past 12 months. Descriptive analysis was conducted using chi-square tests to determine differences between women who reported $\mathrm{AI}$ in the past 12 months with those who did not. Variables were then assessed by univariate logistic regression. Multivariable logistic regression using the stepwise procedure was performed in order to establish variables that were independently associated with AI. Variables significant at the $\mathrm{p} \leq .1$ level in univariate analysis were entered in the multivariable model. To be retained in the final model, all variables had to be significant at the $\mathrm{p} \leq .05$ level.

Categorical variables were dichotomized to the most relevant categories: less than high school education versus high school diploma/equivalent and higher, estimated annual income less than $\$ 10,000$ versus $\$ 10,000$ or more, sexual identity of heterosexual versus homosexual or bisexual, binge drinking once a month or more versus less than once a month, and exposure to either one-on-one HIV interventions, group HIV interventions, or having received free condoms in the past 12 months versus no exposure to any of the aforementioned.

Continuous variables were dichotomized to provide meaningful interpretations: 3 or more partners in the past 12 months versus less than 3 , age at sexual initiation younger than 15 years versus 15 years or older, and regular HIV testers were defined as those who reported at least two HIV tests in the previous two years. Data were analyzed using Statistical Analysis Software version 9.2 (Cary, NC).

\section{RESULTS}

Between March and October of 2007, the Chicago NHBS HET team screened 1101 respondents, with 866 (79\%) meeting the eligibility criteria. In total, 851 (98\%) of the 866 consented to participate. Of the total $851,412(48 \%)$ were women. An additional five women were excluded from the analysis due to reporting injection drug use within the past 12 months. Among the 407 women included in analysis, 71 $(17 \%)$ reported participating in anal intercourse in the past 12 months. Of the 71 women who reported AI in the past 12 months, $61(86 \%)$ reported that it was unprotected. AI occurred most commonly with main partners $(87 \%)$, and slightly less than a quarter $(21 \%)$ of women who reported AI in the past 12 months reported that it was with a casual partner (Table 1).

A total of $381(94 \%)$ of women consented to the HIV test; $7(1.84 \%)$ tested HIV positive via Orasure ${ }^{\circledR}$ Western blot. HIV prevalence rates were similar among women who reported AI compared to those who did not report AI $(1.52 \%$ vs $1.9 \%$; Fisher's exact test $\mathrm{p}=1.0$ ).

The mean age of participants was 31.7 years. The majority of women reported being Black/African-American $(82 \%)$, had an estimated annual income less than $\$ 10,000$ $(81 \%)$, and had at least a high school education or equivalent $(66 \%)$. Approximately $15 \%$ were homeless in the past 12 months, and $11 \%$ had been arrested and detained for over 24 hours in the past 12 months (Table 1).

Almost all of the women in the sample reported unprotected vaginal intercourse in the past 12 months $(87 \%)$. Approximately one-third of women reported being younger than 15 years old at first vaginal or anal intercourse $(32 \%)$. About one-tenth of women reported three or more sexual partners in the past 12 months (11\%), and less than ten percent reported exchange sex in the past 12 months $(7 \%)$. Seven percent reported a sexual identity other than heterosexual.

Slightly less than a third of women reported having concurrent partners in the past 12 months (31\%), and $15 \%$ reported being diagnosed with a STI in the past 12 months. Gonorrhea and Chlamydia were the most prevalent STIs, with $12 \%$ of women reporting being diagnosed with either infection in the past 12 months. Trichomoniasis was also common, with $4 \%$ of women reporting diagnosis in the past 12 months.

Only a third of women reported being tested for HIV on a regular basis $(30 \%)$, and $88 \%$ had not been exposed to or utilized any HIV prevention services in the past 12 months. Almost half of all women in the sample reported using an illicit non-injection drug in the past 12 months (42\%), and over a third reported binge drinking at least once a month in the past 12 months (38\%) (Table 1).

Table 2 shows the factors associated with AI in univariate analysis. AI was positively associated with the following behaviors in the past 12 months: homelessness, being arrested and detained for over 24 hours, STI diagnosis in the past 12 months, illicit non-injection drug use, having three or more sexual partners in the past 12 months, and having any exchange sex. 
Table 1. Demographic, Sexual, and Drug Use Behaviors of Heterosexual Women at Increased Risk in Chicago

\begin{tabular}{|c|c|c|}
\hline & $(\#)(n=407)$ & $(\%)$ \\
\hline Had anal intercourse in the past 12 months & 71 & 17 \\
\hline Had anal intercourse with a main partner in the past 12 months & 62 & 15 \\
\hline Had anal intercourse with a casual partner in the past 12 months & 15 & 4 \\
\hline Black & 334 & 82 \\
\hline Hispanic & 62 & 15 \\
\hline Other & 6 & 1 \\
\hline \multicolumn{3}{|l|}{ Age (Years) } \\
\hline $45-50$ & 46 & 11 \\
\hline \multicolumn{3}{|l|}{ Estimated Annual Income (\$) } \\
\hline $0-9,999$ & 321 & 81 \\
\hline $10,000+$ & 75 & 19 \\
\hline Unemployed & 228 & 57 \\
\hline \multicolumn{3}{|l|}{ Highest Level of Education } \\
\hline Less than high-school degree & 136 & 33 \\
\hline High-school degree & 144 & 35 \\
\hline Binge drinking $\geq$ once a month in past 12 months & 155 & 38 \\
\hline Unprotected vaginal intercourse in the past 12 months & 356 & 87 \\
\hline Concurrency during last sexual relationship & 123 & 31 \\
\hline STI diagnosis in the past 12 months* & 62 & 15 \\
\hline Syphilis & 2 & $<1$ \\
\hline Gonorrhea & 18 & 4 \\
\hline Chlamydia & 33 & 8 \\
\hline Herpes & 6 & 1 \\
\hline Human papillomavirus & 2 & $<1$ \\
\hline Trichomoniasis & 16 & 4 \\
\hline Pubic Lice & 2 & $<1$ \\
\hline Not tested for HIV on a regular basis & 218 & 54 \\
\hline No exposure to or utilization of HIV prevention services & 359 & 88 \\
\hline Age at first vaginal or anal intercourse $<15$ years & 132 & 32 \\
\hline$\geq 3$ sex partners in the past 12 months & 45 & 11 \\
\hline Any exchange sex in the past 12 months & 30 & 7 \\
\hline
\end{tabular}

Not all categories sum to 407 due to missing data.

* 13 women reported more than 1 type of STI diagnosis in the past 12 months 
Table 2. Bivariate Analysis of Demographic, Sexual, and Drug Use Behaviors Among Heterosexual Women at Increased Risk in Chicago by Anal Intercourse in the Past 12 Months

\begin{tabular}{|c|c|c|c|}
\hline & $\begin{array}{c}\text { Anal } \\
(n=71) \\
N(\%)\end{array}$ & 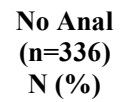 & $\begin{array}{c}\text { OR } \\
(95 \% \mathrm{CI})\end{array}$ \\
\hline $18-24$ & $19(27)$ & $104(31)$ & Referent \\
\hline $25-34$ & $19(27)$ & $99(29)$ & $1.05(.53-2.10)$ \\
\hline $45-50$ & $9(13)$ & $37(11)$ & $1.33(.55-3.2)$ \\
\hline \multicolumn{4}{|l|}{ Race/Ethnicity } \\
\hline Black & $55(77)$ & $279(83)$ & Referent \\
\hline Hispanic & $12(17)$ & $50(15)$ & $.84(.38-1.88)$ \\
\hline Homeless in the past 12 months*** & $20(28)$ & $40(12)$ & $2.90(1.57-5.36)$ \\
\hline Unemployed & $42(60)$ & $186(56)$ & $1.18(.70-1.99)$ \\
\hline Arrested in the past 12 months** & $14(20)$ & $31(9)$ & $2.42(1.21-4.83)$ \\
\hline Concurrency during last sexual relationship**** & $34(50)$ & $89(27)$ & $2.69(1.58-4.59)$ \\
\hline Unprotected vaginal intercourse in the past 12 months & $66(93)$ & $290(86)$ & $2.09(.80-5.47)$ \\
\hline Any non-injection drug use $e^{* * *}$ & $43(61)$ & $128(38)$ & $2.49(1.48-4.22)$ \\
\hline Not tested for HIV on a regular basis & $53(75)$ & $232(69)$ & $1.29(.72-2.32)$ \\
\hline No exposure to or utilization of HIV prevention services & $65(92)$ & $294(88)$ & $1.56(.63-3.79)$ \\
\hline
\end{tabular}

$\mathrm{CI}=$ confidence interval, $\mathrm{OR}=$ odds ratio

$* * .01 \leq p \leq .05 * * * p \leq .01$.

${ }^{a}$ Fisher's exact test used where expected cell value was less than 5 . Confidence intervals cannot be calculated.

AI was also positively associated with having less than a high school education, sexual identity of homosexual or bisexual, having concurrent partners, and having vaginal or anal intercourse for the first time before the age of 15 . AI was not associated with age, race, estimated annual income, unemployment, binge drinking at least once a month, unprotected vaginal intercourse in the past 12 months, not testing for HIV on a regular basis, and not being exposed to or utilizing HIV prevention services in the past 12 months.

The results of multivariable logistic regression are shown in Table 3. The model was adjusted for age and having had unprotected vaginal intercourse in the past 12 months. Three variables were independently correlated with anal intercourse in the past 12 months: having three or more sex partners in the past 12 months (OR 3.27, 95\% CI 1.53-6.99), self-reported STI diagnosis in the past 12 months (OR 2.13,
95\% CI 1.06-4.26), and having had vaginal or anal intercourse for the first time before the age of 15 (OR 2.23, 95\% CI 1.28-3.89). The Hosmer-Lemeshow goodness-of-fit test statistic indicates good model fit $(p=.95)$.

\section{DISCUSSION}

We found that $17 \%$ of women at increased risk in Chicago reported AI in the previous 12 months. The majority $(86 \%)$ of the women who reported AI in the previous 12 months reported that it was unprotected. Consequently, the exploration of factors associated with anal intercourse aids in the identification and subsequent targeting of heterosexual women who are most susceptible to HIV acquisition.

Women in our study who had AI in the past 12 months were more than three times as likely to report having three or more sexual partners in the past 12 months than women who only had 
vaginal intercourse. The relationship between multiple sex partners and AI found in our sample is consistent with previous literature $[8,16,17]$.

Table 3. Multivariable Logistic Regression: Factors Associated with Anal Intercourse Among Heterosexual Women at Increased Risk in Chicago

\begin{tabular}{|c|c|c|c|}
\hline & OR & $95 \%$ CI & Wald Test $\mathbf{P}$ \\
\hline \multicolumn{4}{|l|}{ Age (Years) } \\
\hline $18-24$ & Referent & & \\
\hline $25-34$ & 1.01 & $(.49-2.09)$ & .972 \\
\hline $35-44$ & 1.20 & $(.58-2.48)$ & .615 \\
\hline $45-50$ & 1.41 & $(.56-3.55)$ & .472 \\
\hline \multicolumn{4}{|c|}{ Unprotected Vaginal Intercourse in the Past 12 Months } \\
\hline No & Referent & & \\
\hline Yes & 1.77 & $(.64-4.88)$ & .272 \\
\hline \multicolumn{4}{|c|}{$\geq 3$ Sex Partners in the Past 12 Months } \\
\hline No & Referent & & \\
\hline Yes & 3.27 & $(1.53-6.99)$ & .002 \\
\hline \multicolumn{4}{|c|}{ STI Diagnosis in the Past 12 Months } \\
\hline No & Referent & & \\
\hline Yes & 2.13 & $(1.06-4.26)$ & .033 \\
\hline \multicolumn{4}{|c|}{ Age at First Vaginal or Anal Intercourse $<15$ Years } \\
\hline No & Referent & & \\
\hline Yes & 2.23 & $(1.28-3.89)$ & .005 \\
\hline
\end{tabular}

Having multiple sex partners has been independently linked to HIV transmission [18]; the synthesis of having multiple sex partners and AI may compound this risk. It has been proposed that women who engage in anal intercourse have a tendency to participate in other behaviors that put them at risk for HIV [19, 20]. The combination of risk factors associated with AI in our sample further highlights the importance of targeting this group.

Data on the relationship between STI diagnosis and anal intercourse are inconsistent. A study by Gross et al. of women at-risk for HIV infection found that self-reported STI diagnosis was associated with AI [21]. In contrast, three studies measuring the correlation between laboratory confirmed STI diagnosis and anal intercourse did not find such associations [8, 9, 22]. Our findings coincide with those of Gross et al. [21].

Several women in our study reported being diagnosed with a STI in the past year. The majority of the STIs reported were non-ulcerative. Non-ulcerative STIs have been noted to increase the risk of HIV transmission, particularly from the insertive to the receptive partner [23]. Women in our sample who reported having $\mathrm{AI}$ in the past 12 months had a 2.13 increase in odds of reporting a STI diagnosis in the past 12 months, after controlling for unprotected vaginal intercourse. This association indicates that STI clinic interventions which emphasize consistent condom use during AI may be more effective than those that address unprotected vaginal intercourse alone.

Our study also found that women who had vaginal or anal intercourse for the first time before the age of 15 were more than twice as likely to report AI in the past 12 months compared to women who initiated intercourse at a later age. Few studies have measured age at sexual initiation as a correlate of AI. Baldwin and Baldwin found that among a random sample of U.S. university students, $33.1 \%$ who participated in AI had vaginal intercourse at or before the age 15 , whereas only $20.8 \%$ of those who did not participate AI had vaginal intercourse at or before the age 15 [19].

The noted association between AI and early sexual initiation in samples with different demographic characteristics suggests a strong relationship between these factors. Informing young sexually active women or women who report sexual activity at a young age about the risks associated with anal intercourse may prove to be beneficial with regard to STI/HIV reduction.

The overall prevalence of sexual initiation before the age of 15 in our sample was high (32\%) compared to general estimates of this behavior. Data from the National Survey of Family Growth show that only $13.5 \%$ of non-Hispanic Black females and $5.9 \%$ of non-Hispanic White females initiate sexual intercourse before the age of 15 [23]. This finding signals a need for interventions that incorporate strategies on building selfconfidence and developing relationship negotiation skills for adolescent females living in poverty.

There are limitations to this study. This study was crosssectional so causality between the HIV risk factors and AI cannot be determined. These data are not generalizable to all urban poverty areas or non-urban poverty areas. All of the data were self-reported so sensitive risk factors, including the outcome variable, may have been underreported. The data used to identify HRAs was limited to AIDS case surveillance data. Chicago HIV case surveillance data as of 2004 was unable to be geocoded due to limitations of code based reporting. Lastly, these data have not been weighted by venue selection probability.

\section{CONCLUSION}

The high prevalence of HIV and the low rate of prevention utilization in our sample clearly demonstrate a need for enhanced prevention strategies which target heterosexual women at increased risk. The identification of factors associated with anal intercourse may be an important first step. However, addressing these factors without considering the social context in which they occur would be short-sighted.

The rates of homelessness, unemployment, low educational attainment, and incarceration among study participants were extremely high. A better understanding of how these structural factors affect risk behaviors such as anal intercourse could enhance HIV prevention and health promotion efforts in the future.

Efforts to mitigate structural factors related to HIV and STIs require the coordination and integration of resources from multiple disciplines. Attributes of potential interventions may include economic skill-building, development of sexual negotiation skills in women, expansion of constructive youth social programs, and strategies which reduce social instability and residential isolation. 


\section{ACKNOWLEDGEMENTS}

The authors would like to extend their gratitude to all Chicago NHBS research participants. We would also like to thank the Chicago NHBS data collection staff, Diane Clay, Thomas Clyde, Nayda DeLaRosa, and Edgar Gutierrez for their tireless efforts. Thank you also to Elizabeth DiNenno, our CDC project officer during NHBS HET, for her guidance and support.

Funding for this study and analysis was provided by the Centers for Disease Control and Prevention under Program Announcement 04017/Cooperative Agreement U62/CCU523 584.

\section{CONFLICT OF INTEREST}

The authors confirm that this article content has no conflict of interest.

\section{REFERENCES}

[1] Denning P, DiNenno E. Communities in crisis: is there a generalized HIV epidemic in impoverished urban areas of the United States? International AIDS Conference 2010; Abstract WEPDD103, 18-23 July, Vienna Austria.

[2] Centers for Disease Control and Prevention. HIV Surveillance Report, 2008; 20. Available from http://www.cdc.gov/hiv/topics/ surveillance/resources/reports [Accessed on November 15th 2010].

[3] Chicago Department of Public Health, STI/HIV/AIDS Chicago, Surveillance Repot. Division of Chicago Department of Public Health, Fall 2010. Available from http://www.cityofchicago. org/city/en/depts/cdph/provdrs/sti_hiv_aids.html [Accessed on December 13th, 2010]

[4] Mosher WD, Chandra A, Jones J. Sexual Behavior and Selected Health Measures: Men and Women 15-44 Years of Age, United States, 2002. Advance data from vital and health statistics; no 362, Hyattsville, MD: National Center of Health Statistics 2005: pp. 156.

[5] Laumann EO. Gagnon JH, Michael RT, Michaels S. The Social Organization of Sexuality: Sexual Practices in the United States. $1^{\text {st }}$ ed. USA: University of Chicago Press 1994.

[6] Varghese B, Maher JE, Peterman TA, Branson BM, Steketee RW. Reducing the risk of sexual HIV transmission quantifying the peract risk for HIV on the basis of choice of partner, sex act and condom use. Sex Transm Dis 2002; 29: 38-43.

[7] Halperin DT. Heterosexual anal intercourse: prevalence, cultural factors, and HIV infection and other health risks, Part 1. AIDS Patient Care STDs 1999; 13: 717-30.

[8] Gorbach PM, Manhart LE, Hess KL, Stoner BP, Martin DH, Holmes KK. anal intercourse among young heterosexuals in three sexually transmitted disease clinics in the United States. Sex Transm Dis 2009; 36: 193-8.
[9] Javanbakht M, Guerry S, Gorbach PM, et al. prevalence and correlates of heterosexual anal intercourse among clients attending public sexually transmitted disease clinics in Los Angeles county. Sex Transm Dis 2010; 37: 369-76.

[10] Mackesy-Amiti ME, McKirnin DJ, Ouellet LJ. Relationship characteristics associated with anal sex among female drug users. Sex Transm Dis 2010; 37: 346-51.

[11] Reynolds GL, Latimore AD, Fisher DG. Heterosexual anal sex among female drug users: U.S. national compared to local long beach, California data. AIDS Behav 2008; 12: 796-805.

[12] Karon JM, Fleming PL, Steketee RW, DeCock KM. HIV in the United States at the turn of the century: an epidemic in transition. Am J Public Health 2001; 91: 1060-8.

[13] Peterman TA, Lindsey CA, Selik RM. This place is killing me: a comparison of counties where the incidence rates of AIDS increased the most and the least. J Infect Dis 2005; 191 (Suppl 1): S123-6.

[14] MacKellar DA, Gallagher KM, Finlayson T, Sanchez T, Lansky A, Sullivan PS. Surveillance of HIV risk and prevention behaviors of men who have sex with men- a national application of venuebased, time-space sampling. Public Health Rep 2007; 122 (Suppl 1): $39-47$.

[15] National Institute of Alcohol Abuse and Alcoholism. NIAAA council approves definition of binge drinking. NIAAA Newsletter 2004; No. 3, p. 3. Available from http://pubs.niaaa.nih.gov/publica tions/Newsletter/winter2004/Newsletter_Number3.pdf. [Accessed on April 3rd, 2011].

[16] Lescano CM, Houck CD, Brown LK, et al. Correlates of heterosexual anal intercourse among at-risk adolescents and young adults. Am J Public Health 2009; 99: 1131-6.

[17] Tian LH, Peterman TA, Tau G, et al. Heterosexual anal sex activity in the year after an std clinic visit. Sex Transm Dis 2008; 35: 9059.

[18] Piot P, Taelman H, Minlangu KB, et al. Acquired immunodeficiency syndrome in a heterosexual population in Zaire. Lancet 1984; 2: 65-9.

[19] Baldwin JI, Baldwin JD. Heterosexual anal intercourse: an understudied, high-risk sexual behavior. Arch Sex Behav 2000; 29 : 357-73.

[20] Erikson PI, Bastani R, Maxwell AE, Marcus AC, Capell FJ, Yan KX. Prevalence of anal sex among heterosexuals in California and its relationship to other AIDS risk behaviors. AIDS Educ Prev 1995; 7: 477-93.

[21] Gross M, Holte SE, Marmor M, Mwatha A, Koblin BA, Mayer $\mathrm{KH}$. Anal sex among HIV-seronegative women at high risk of HIV exposure. J Acq Immun Def Synd 2000; 24: 393-8.

[22] Misegades L, Page-Shafer K, Halperin D, McFarland W. Anal intercourse among low-income women in California: an overlooked risk factor for HIV? AIDS 2001; 15: 534-5.

[23] Fleming DT, Wasserheit JN. From epidemiological synergy to public health policy and practice: the contribution of other sex transm dis to sexual transmission of HIV infection. Sex Transm Infect 1999; 75: 3-17. 